\title{
INCIDÊNCIA DA PESCA DE ARRASTO CAMAROEIRO SOBRE PEIXES EM ATIVIDADE REPRODUTIVA: UMA AVALIAÇÃO NO LITORAL NORTE DE SANTA CATARINA, BRASIL.
}

\author{
JULIANA VENTURA DE PINA ${ }^{1,2} \&$ PAULO DE TARSO CHAVES ${ }^{1,3}$ \\ ${ }^{1}$ Universidade Federal do Paraná -Programa de Pós-Graduação em Ciências Biológicas - Zoologia, C.P. 19020, 81531-980, Curitiba, Brasil. \\ ²Bolsistas CAPES - julianavpina@hotmail.com; 3 - ptchaves@ufpr.br. e CNPq.
}

\begin{abstract}
As consequências da mudança do período de defeso da pesca do camarão sete-barbas sobre a atividade reprodutiva dos peixes que compõem a captura incidental foram avaliadas através de amostragens numa comunidade no norte de Santa Catatina entre outubro de 2005 e março de 2007. Das 72 espécies reconhecidas, 21\% eram Sciaenidae. O arrasto incidiu predominantemente sobre peixes imaturos, sendo que em 22 espécies apenas indivíduos nessa condição foram registrados. Noutras 32 espécies registraram-se indivíduos maduros, em nove das quais também indivíduos desovados ou espermiados. O Índice de Atividade Reprodutiva apontou que Pellona harroweri e Stellifer sp integram o arrasto em atividade reprodutiva muito intensa, e Isopisthus parvipinnis, Paralonchurus brasiliensis, Stellifer brasiliensis, S. stellifer e Symphurus tesselatus no máximo na categoria intensa. Primavera de 2005, o verão de 2006 e o verão de 2007 foram as estações com espécies em atividade reprodutiva muito intensa. O verão de 2007, tendo sucedido o período de defeso, apresentou apenas uma espécie a mais com atividade reprodutiva intensa que o verão de 2006. Propõe-se, assim, que a mudança da interdição do arrasto, do outono em 2005 para a primavera em 2006, não trouxe reflexos sobre a atividade reprodutiva da ictiofauna na comunidade estudada.
\end{abstract}

RESUMO

PALAVRAS CHAVE. Captura incidental; Índice de Atividade Reprodutiva; reprodução; ictiofauna.

\section{ABSTRACT}

Effects of the shrimp trawling fisheries on the reproductive activity of fish: a study case in southern Brazil.

The consequences of changing in closed seasons of small scale shrimp in Southern Brazil trawling on the breeding activity of fish bycatch species were investigated from samples collected from October 2005 to March 2007. From the 72 species recognized, 21\% were Sciaenidae. Trawling caught mainly immature fish, and individuals of 22 species were found at this condition only. Other 32 species presented mature individuals, nine of them showing also spawned or ripe individuals. Reproductive Activity Index has indicated that in trawling Pellona harroweri and Stellifer $s p$ are found in a very intense breeding activity, and that Isopisthus parvipinnis, Paralonchurus brasiliensis, Stellifer brasiliensis, S. stellifer and Symphurus tesselatus are found in intense breeding activity. Spring 2005, Summer 2006 and Summer 2007 were periods with species in a very intense reproductive activity. Summer 2007, having succeeded the closed season of spring 2006, has showed just one more species in breeding activity than summer 2006, a year when trawling was closed in autumn. In view of this, it is proposed that this change in the closed season for trawling did not affected the reproducitive activity of the fish assemblage as a whole.

KEY WORDS. By-catch; Reproductive Activity Index; breeding; fish assemblage.

\section{INTRODUÇÃO}

A pesca de camarão tem importância econômica em todo o litoral brasileiro (Isaac et al. 2006). No norte de Santa Catarina a espécie-alvo é o camarão sete-barbas, Xiphopenaeus kroyeri, o segundo pescado em volume desembarcado pela frota artesanal e o décimo (mas o primeiro dentre os invertebrados) pela industrial (Sunyé 2006). A pescaria do camarão sete-barbas utiliza rede de arrasto de fundo com portas ou pranchas (Robert \& Chaves 2006). Esta, por ser um petrecho com baixa seletividade, captura um volume de outros invertebrados e de peixes que normalmente supera aquele da espécie-alvo (Branco \& Verani 2006), o que gera uma captura incidental que em sua maior parte é descartada (Chaves et al. 2003).

Na comunidade de Enseada, município de São Francisco do Sul, Souza \& Chaves (2007) avaliaram a condição reprodutiva dos peixes capturados incidentalmente e constataram que metade das espécies apresenta indivíduos que em alguma época do ano são capturados com gônadas maduras. Além disso, os autores observaram que a proibição legal de arrasto camaroeiro na primavera do ano 2006 teve reflexos positivos para a atividade reprodutiva da ictiofauna na estação subsequente. De fato, Arendse et al. (2007) citam que, em se tratando de espécies que para desova formam agregados, como o fazem os Sciaenidae e outros peixes demersais comuns na região (Chaves et al. 2003, Robert \& Chaves 2006), a interrupção da pesca é eficaz ao êxito reprodutivo. 0 presente trabalho realiza uma análise de natureza semelhante àquela de Souza \& Chaves (2007), abordando a ictiofauna dos arrastos camaroeiros da comunidade de Itapema do Norte, município de Itapoá, para avaliar se a seleção do período de defeso do arrasto do camarão sete-barbas tem reflexos sobre a atividade reprodutiva da ictiofauna que $o$ acompanha. 


\section{MATERIAL E MÉTODOS}

Amostragens de desembarque da pesca de arrasto camaroeiro foram realizadas mensalmente, de outubro de 2005 a março de 2007 - exceto no período de defeso de arrasto, ou seja, outubro a dezembro de 2006 - na comunidade de Itapema do Norte, município de Itapoá, litoral norte de Santa Catarina (Figura 1). A pesca é realizada na plataforma continental rasa (profundidade 2 a 10 metros) utilizando redes com pranchas ou portas e malha de $2 \mathrm{~cm}$ entre nós opostos do ensacador. As pescarias são de caráter sol-a-sol, isto é, os pecadores partem na aurora e retornam no mesmo dia. A amostra do mês constou do conjunto de peixes capturados no último arrasto efetuado no dia. Os peixes - total de 20197 - foram acondicionados em isopor com gelo e transportados para laboratório, sendo identificados e mensurados (comprimento total - CT e peso total - PT) (Tabela 1).

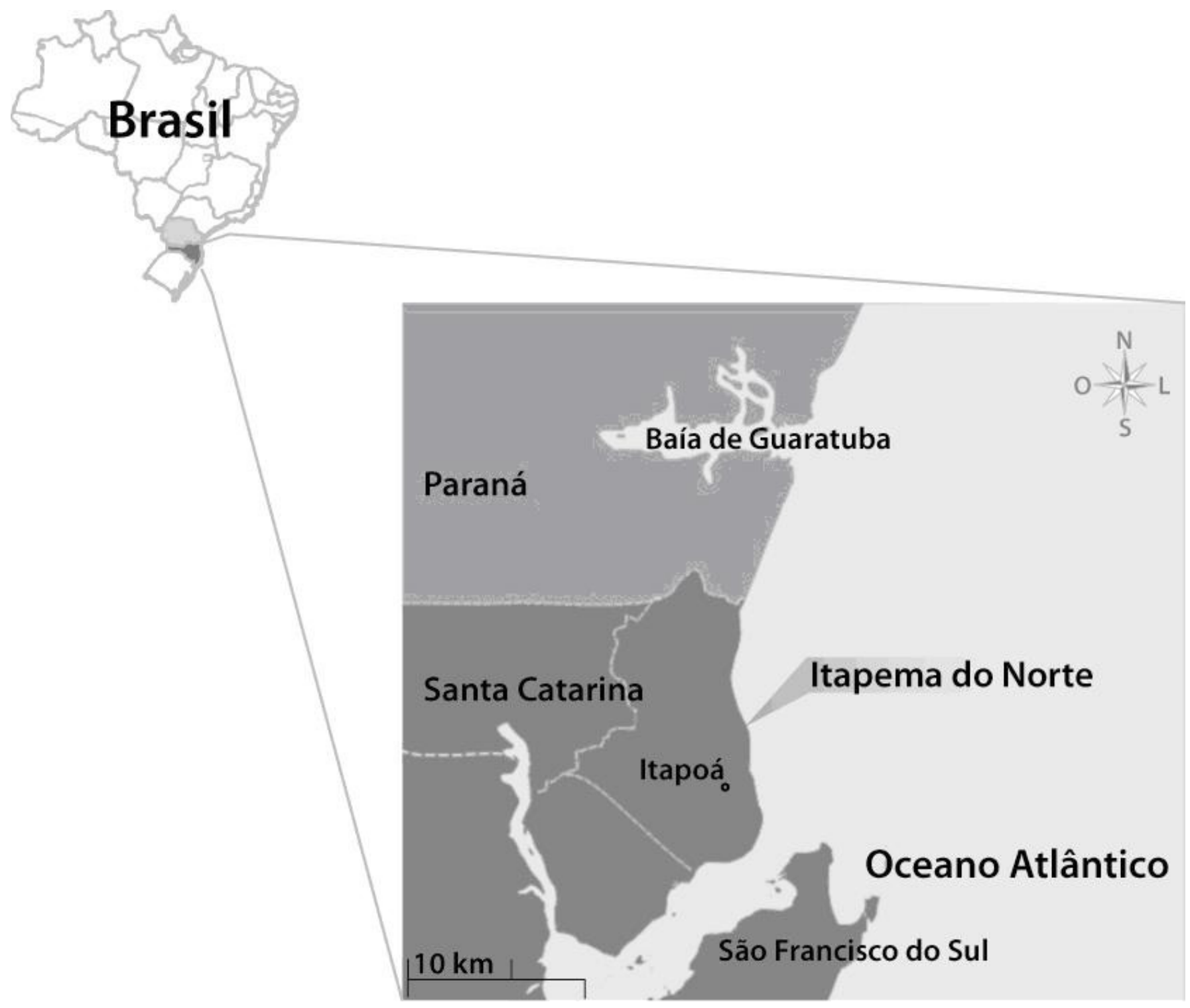

Figura 1 - Localização da comunidade de Itapema do Norte no litoral norte de Santa Catarina, Brasil. 
Tabela 1 - Data de amostragem, número total e peso total de peixes amostrados mensalmente em Itapema do Norte, no período de outubro de 2005 a março de 2007.

\begin{tabular}{ccc}
\hline $\begin{array}{c}\text { Data de } \\
\text { amostragem }\end{array}$ & $\begin{array}{c}\text { Número total } \\
\text { de peixes }\end{array}$ & $\begin{array}{c}\text { Peso total } \\
\text { de peixes }(\boldsymbol{g})\end{array}$ \\
\hline 9/10/2005 & 1033 & 10386,65 \\
$13 / 11 / 2005$ & 1849 & 16438,98 \\
$14 / 12 / 2005$ & 790 & 8620,85 \\
$14 / 01 / 2006$ & 2008 & 13651,94 \\
$12 / 02 / 2006$ & 1034 & 9145,09 \\
$10 / 03 / 2006$ & 1456 & 10819,94 \\
$20 / 04 / 2006$ & 1398 & 10123,64 \\
$20 / 05 / 2006$ & 2002 & 13672,06 \\
$11 / 06 / 2006$ & 1358 & 9956,36 \\
$15 / 07 / 2006$ & 1486 & 8478,01 \\
$12 / 08 / 2006$ & 1211 & 7825,26 \\
$17 / 09 / 2006$ & 971 & 8954,87 \\
$11 / 01 / 2007$ & 1286 & 11425,09 \\
$13 / 02 / 2007$ & 1136 & 12323,04 \\
$16 / 03 / 2007$ & 1179 & 13928,14 \\
\hline TOTAL & 20197 & 165749,92 \\
\hline
\end{tabular}

A constância de cada espécie nas amostras foi calculada pela relação de Dajoz (1983): C= 100.p/P, sendo "p" o número de amostras contendo a espécie em questão e "P" o número total de amostras obtidas. Em função do valor de " $\mathrm{C}$ " as espécies foram consideradas constantes - presentes em mais que $50 \%$ das amostras, acessórias - presentes entre $25 \%$ e $50 \%$ das amostras, ou acidentais - presentes em menos que $25 \%$ das amostras.

Para a análise da reprodução, nas espécies com $\mathrm{n} \leq 30$, todos os indivíduos foram trabalhados; já nas espécies com $n>30$ foi realizada uma subamostragem, contemplando um total de 30 indivíduos por arrasto, distribuídos em pequeno, médio e grande portes. O sexo dos exemplares foi reconhecido e o estádio de maturação gonadal classificado baseandose em Vazzoler (1996): imaturos - A, em maturação $\mathrm{B}$, maduros - C e desovados (fêmeas) ou espermiados (machos) - D. Na sequência as gônadas foram retiradas e pesadas.

Os indicadores da atividade reprodutiva consideraram: (i) a frequência de estádios de maturação gonadal; (ii) os valores de IGS - índice gonadossomático, calculado individualmente como IGS= 100.PG/PT, sendo PG o peso das gônadas; no tratamento de IGS por conjunto de indivíduos apenas aqueles adultos (estádios B, C, ou D) foram utilizados; e (iii) IAR - Índice de Atividade Reprodutiva (Agostinho et al. 1993; exclusivo para fêmeas). Segue a fórmula:

$$
\operatorname{IAR}_{S p_{x, k} K}=\frac{\ln N_{i}\left[\frac{n_{i}}{\sum_{j: 1 \rightarrow 5} n_{i, j}}+\frac{n_{i}}{N_{i}}\right] \frac{I G S_{i}}{I G S_{e}}}{\ln N_{m}\left[\frac{n_{m}}{\sum_{j: 1 \rightarrow 5} n_{i, j}}+1\right]} 100
$$

onde: $\mathrm{N}_{\mathrm{i}}$ O número de indivíduos na unidade amostral (u.a.) "”, ou seja, o número de indivíduos da $\mathrm{sp}_{\mathrm{x}}$ na unidade amostral $\mathrm{K}, \mathrm{sp}_{\mathrm{x}}$ é a espécie em questão, $\mathrm{K}$ é a unidade amostral em questão, $n_{i} 0$ número de indivíduos em reprodução na u.a. "”, $\mathrm{N}_{\mathrm{m}} \mathrm{O}$ número de indivíduos na u.a. com maior número de peixes da $\mathrm{sp}_{\mathrm{x}}$, considerando o conjunto das cinco unidades amostrais (j), $\mathrm{n}_{\mathrm{m}}$ o número de indivíduos em reprodução (estádios $\mathrm{C}$ e/ou $\mathrm{D}$ ) na u.a. com maior número de peixes da $\mathrm{sp}_{\mathrm{x}}$, considerando o conjunto das cinco unidades amostrais (j), IGS $\mathrm{S}_{\mathrm{i}}$ o valor médio de IGS dos indivíduos em reprodução na u.a. "i" e $\mathrm{IGS}_{\mathrm{e}} \mathrm{O}$ valor individual mais alto de IGS, considerando o conjunto das unidades amostrais (j).

Os índices IGS e IAR foram aplicados apenas 
às espécies representadas por mais que 30 indivíduos e mínimo de cinco indivíduos em pelo menos duas estações amostradas, o que permitiu comparações. Além disso, uma vez que a aplicação do IAR objetivava distinguir diferentes graus de atividade reprodutiva entre espécies apresentando fêmeas maduras ou pós-desova, e considerando-se a restrição criada pela presença de logaritmo na fórmula, nas situações em que $\mathrm{n}_{\mathrm{i}}=0$ esse Índice não foi aplicado. Ainda seguindo-se Agostinho et al. (1993), a atividade reprodutiva das espécies foi classificada em nula (IAR $\leq 2$ ou não aplicado em função de $\left.\mathrm{n}_{\mathrm{i}}=0\right)$, incipiente $(2<\mid \mathrm{A} R \leq 5)$, moderada $(5<\mid A R \leq 10)$, intensa $(10<\mid A R \leq 20)$ e muito intensa $(I A R>20)$. Os dados foram agrupados em estações do ano, correspondendo outubro, novembro e dezembro à primavera e assim sucessivamente.

\section{RESULTADOS}

No conjunto das amostras registraram-se 72 espécies, além de um número não determinado de outras do gênero Cynoscion, nas quais o pequeno tamanho impossibilitou a identificação nesse nível. Das 31 famílias, as mais representativas em espécies foram Sciaenidae (14 espécies, 21\%), Engraulidae (sete espécies, 10\%) e Carangidae (cinco espécies, $7 \%$ ) (Tabela 2). A maioria das espécies (31, correspondendo a $43 \%$ do total) ocorreu com frequência classificada como acidental. Outras 28 espécies (39\%) foram constantes e 13 (18\%), acessórias. Das espécies constantes, seis estiveram presentes em todas as amostragens: Isopisthus parvipinnis, Paralonchurus brasiliensis, Pellona harroweri, Stellifer brasiliensis, Stellifer rastrifer e Symphurus tessellatus. Dezessete espécies ocorreram uma única vez, 14 das quais com apenas um indivíduo (Tabela 2).

Tabela 2 - Relação das espécies de peixes amostradas nas pescarias de arrasto, com suas abundância absoluta, frequência relativa $(\mathrm{F} \%)$, valor percentual de constância $(\mathrm{C})$, intervalos de comprimento, número de peixes subamostrados com seus estádios de maturação gonadal, no período de outubro de 2005 a março de 2007. Negrito: espécies em que ocorreram todos os estádios de maturação.

\begin{tabular}{|c|c|c|c|c|c|c|c|c|c|c|c|}
\hline FAMÍLIA & ESPÉCIE & $\begin{array}{c}N \\
\text { amostrado }\end{array}$ & $F \%$ & $C$ & $\begin{array}{c}C T \\
\text { Min } \\
(\mathbf{m m})\end{array}$ & $\begin{array}{c}C T \\
\text { Max } \\
(\mathrm{mm})\end{array}$ & $\begin{array}{c}N \text { sub- } \\
\text { amostrado }\end{array}$ & $\boldsymbol{A}$ & B & $C$ & $D / E$ \\
\hline \multirow[t]{4}{*}{ Achiridae } & Achirus declivis Chabanaud, 1940 & 22 & 0,11 & 46,7 & 68 & 190 & 22 & 14 & 2 & 5 & 1 \\
\hline & Achirus lineatus (Linnaeus, 1758) & 9 & 0,04 & 33,3 & 55 & 112 & 8 & 4 & 3 & 1 & 0 \\
\hline & $\begin{array}{l}\text { Trinectes microphthalmus } \\
\text { (Chabanaud, 1928) }\end{array}$ & 26 & 0,13 & 60,0 & 32 & 161 & 21 & 20 & 0 & 1 & 0 \\
\hline & $\begin{array}{l}\text { Trinectes paulistanus (Ribeiro, } \\
\text { 1915) }\end{array}$ & 90 & 0,45 & 73,3 & 62 & 165 & 80 & 57 & 11 & 11 & 1 \\
\hline \multirow[t]{3}{*}{ Ariidae } & Cathorops spixii (Agassiz, 1829) & 285 & 1,41 & 60,0 & 72 & 273 & 240 & 228 & 11 & 1 & 0 \\
\hline & Genidens barbus (Lacepède, 1803) & 6 & 0,03 & 20,0 & 77 & 171 & 6 & 6 & 0 & 0 & 0 \\
\hline & Genidens genidens (Cuvier, 1829) & 12 & 0,06 & 20,0 & 75 & 194 & 12 & 12 & 0 & 0 & 0 \\
\hline \multirow[t]{2}{*}{ Batrachoididae } & $\begin{array}{l}\text { Porichthys porosissimus (Cuvier, } \\
\text { 1829) }\end{array}$ & 9 & 0,04 & 20,0 & 65 & 181 & 9 & 8 & 0 & 1 & 0 \\
\hline & $\begin{array}{l}\text { Thalassophryne nattereri } \\
\text { Steindachner, } 1876\end{array}$ & 1 & 0,00 & 6,67 & 36 & 36 & 1 & 1 & 0 & 0 & 0 \\
\hline \multirow[t]{5}{*}{ Carangidae } & $\begin{array}{l}\text { Chloroscombrus chrysurus } \\
\text { (Linnaeus, 1766) }\end{array}$ & 96 & 0,48 & 66,7 & 30 & 159 & 92 & 86 & 4 & 2 & 0 \\
\hline & $\begin{array}{l}\text { Hemicaranx amblyrhynchus } \\
\text { (Cuvier, 1833) }\end{array}$ & 49 & 0,24 & 46,7 & 50 & 210 & 48 & 47 & 1 & 0 & 0 \\
\hline & $\begin{array}{l}\text { Oligoplites saurus (Bloch \& } \\
\text { Schneider, 1801) }\end{array}$ & 45 & 0,22 & 53,3 & 45 & 213 & 45 & 45 & 0 & 0 & 0 \\
\hline & Selene setapinnis (Mitchill, 1815) & 360 & 1,78 & 73,3 & 35 & 187 & 215 & 215 & 0 & 0 & 0 \\
\hline & Selene vomer (Linnaeus, 1758) & 33 & 0,16 & 60,0 & 31 & 110 & 30 & 30 & 0 & 0 & 0 \\
\hline Centropomidae & $\begin{array}{l}\text { Centropomus undecimalis (Bloch, } \\
\text { 1792) }\end{array}$ & 1 & 0,00 & 6,67 & 239 & 239 & 1 & 0 & 0 & 1 & 0 \\
\hline Clupeidae & $\begin{array}{l}\text { Opisthonema oglinum (Lesueur, } \\
\text { 1818) }\end{array}$ & 15 & 0,07 & 13,3 & 82 & 114 & 32 & 24 & 8 & 0 & 0 \\
\hline Cynoglossidae & $\begin{array}{l}\text { Symphurus tessellatus (Quoy \& } \\
\text { Gaimard, 1824) }\end{array}$ & 300 & 1,49 & 100 & 54 & 170 & 292 & 273 & 18 & 1 & 0 \\
\hline Dactylopteridae & $\begin{array}{l}\text { Dactylopterus volitans (Linnaeus, } \\
\text { 1758) }\end{array}$ & 2 & 0,01 & 13,3 & 143 & 191 & 2 & 1 & 1 & 0 & 0 \\
\hline Diodontidae & $\begin{array}{l}\text { Chilomycterus spinosus spinosus } \\
\text { (L.innaeus, 1758) }\end{array}$ & 2 & 0,01 & 6,67 & 30 & 80 & 2 & 2 & 0 & 0 & 0 \\
\hline
\end{tabular}




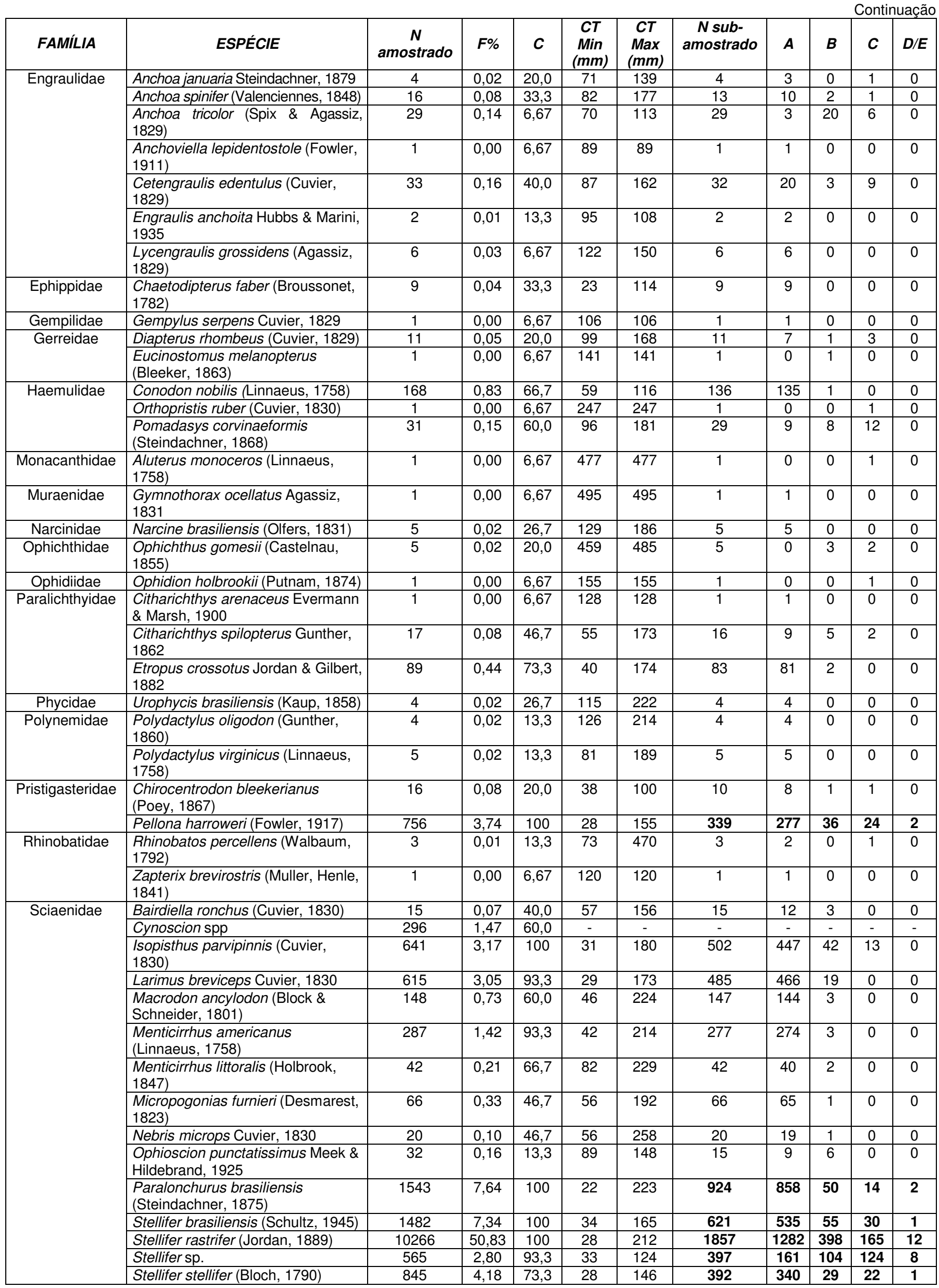




\begin{tabular}{|c|c|c|c|c|c|c|c|c|c|c|c|}
\hline FAMÍLIA & ESPÉCIE & $\underset{\text { amostrado }}{N}$ & $F \%$ & $C$ & $\begin{array}{c}C T \\
\text { Min } \\
(\mathrm{mm})\end{array}$ & $\begin{array}{c}C T \\
\operatorname{Max} \\
(\mathrm{mm})\end{array}$ & $\begin{array}{c}N \text { sub- } \\
\text { amostrado }\end{array}$ & $\boldsymbol{A}$ & B & $C$ & $D / E$ \\
\hline Scombridae & $\begin{array}{l}\text { Scomberomorus brasiliensis } \\
\text { Collette, Russo \& Zavala-Camim, } \\
1978\end{array}$ & 2 & 0,01 & 13,3 & 180 & 206 & 2 & 2 & 0 & 0 & 0 \\
\hline \multirow[t]{3}{*}{ Serranidae } & $\begin{array}{l}\text { Diplectrum radiale (Quoy \& } \\
\text { Gaimard, 1824) }\end{array}$ & 1 & 0,00 & 6,67 & 119 & 119 & 1 & 1 & 0 & 0 & 0 \\
\hline & Rypticus randalli Courtenay, 1967 & 11 & 0,05 & 46,7 & 87 & 131 & 11 & 3 & 5 & 3 & 0 \\
\hline & Serranus phoebe Poey, 1851 & 1 & 0,00 & 6,67 & 112 & 112 & 1 & 0 & 1 & 0 & 0 \\
\hline Stromateidae & Peprilus paru (Linnaeus, 1758) & 70 & 0,35 & 66,7 & 24 & 185 & 63 & 62 & 1 & 0 & 0 \\
\hline \multirow[t]{3}{*}{ Tetraodontidae } & $\begin{array}{l}\text { Lagocephalus laevigatus } \\
\text { (Linnaeus, 1766) }\end{array}$ & 436 & 2,16 & 93,3 & 43 & 173 & 366 & 364 & 2 & 0 & 0 \\
\hline & Sphoeroides greeleyi Gilbert, 1900 & 1 & 0,00 & 6,67 & 124 & 124 & 1 & 0 & 1 & 0 & 0 \\
\hline & $\begin{array}{l}\text { Sphoeroides testudineus } \\
\text { (Linnaues, 1758) }\end{array}$ & 34 & 0,17 & 53,3 & 24 & 260 & 32 & 7 & 13 & 11 & 1 \\
\hline Trichiuridae & Trichiurus lepturus Linnaeus, 1758 & 168 & 0,83 & 80,0 & 92 & 771 & 140 & 132 & 5 & 3 & 0 \\
\hline Triglidae & Prionotus punctatus (Bloch, 1793) & 25 & 0,12 & 53,3 & 31 & 119 & 24 & 24 & 0 & 0 & 0 \\
\hline TOTAL & & 20197 & 100 & & & & 8313 & 6924 & 886 & 474 & 29 \\
\hline
\end{tabular}

A pesca camaroeira incidiu predominantemente sobre peixes imaturos, correspondendo a 6924 indivíduos de 64 espécies. O estádio em maturação foi registrado em 886 indivíduos de 42 espécies; o estádio maduro, em 474 indivíduos de 32 espécies; e o estádio desovado ou espermiado, em 29 indivíduos de nove espécies (Tabela 2). Vinte e duas espécies (83\% do total de indivíduos amostrados) foram registradas apenas no estádio imaturo, mas outras nove evidenciaram sinais de desova na região, visto terem apresentado tanto indivíduos maduros, como desovados ou espermiados. Essas espécies corresponderam a $6 \%$ das amostragens do arrasto (Tabela 2).

Oito espécies atenderam os requisitos para análise de atividade reprodutiva. Duas delas Pellona harroweri e Stellifer $s p$ - foram registradas com atividade reprodutiva muito intensa. Em cinco outras os valores de IAR corresponderam no máximo à categoria intensa: Isopisthus parvipinnis, Paralonchurus brasiliensis, Stellifer brasiliensis, $S$. stellifer e Symphurus tesselatus. E em Stellifer rastrifer no máximo à categoria moderada (Figura 2).

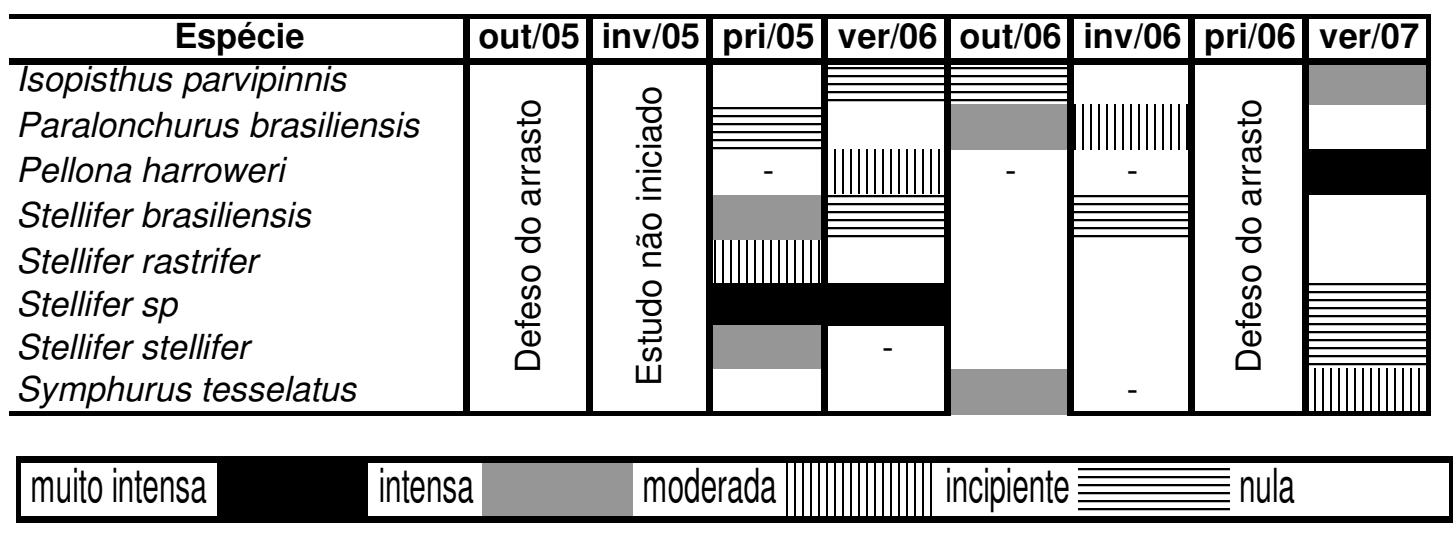

Figura 2 - Distribuição sazonal das categorias de Índice de Atividade Reprodutiva nas espécies, do outono de 2005 (out/05) ao verão de 2007 (ver/07). (-) n<5.

Exceto no inverno, em todas as estações amostradas pelo menos uma espécie encontrava-se em atividade reprodutiva intensa ou muito intensa. A primavera de 2005, o outono de 2006 e o verão de 2007 foram aquelas em que 0 arrasto incidiu sobre número maior (três, duas e duas, respectivamente) de espécies em atividade reprodutiva nessas categorias
(Figura 3). Uma comparação entre os dois verões estudados quanto à atividade reprodutiva das espécies que foram comuns a ambos evidencia que 0 verão de 2007, tendo sucedido imediatamente o período de defeso do arrasto camaroeiro, apresentou uma espécie a mais com maior grau de atividade reprodutiva que o verão de 2006 (Figura 2). Apenas 
duas espécies - $S$. brasilisensis e Stellifer $s p-$ apresentaram-se no verão de 2006 com intensidade reprodutiva maior que no verão 2007.

\section{DISCUSSÃO}

A composição ictiofaunística dos arrastos de camarão na região de estudo pode ser considerada de alta diversificação. No litoral sul do Estado do Paraná, Chaves et al. (2003) e Gomes \& Chaves (2006) registraram sessenta e duas e sessenta e uma espécies, respectivamente, e no litoral norte de Santa Catarina Branco \& Verani (2006) e Souza \& Chaves (2007) sessenta e setenta e seis, respectivamente. No presente estudo, mais de um terço das setenta e duas espécies foi classificada como de presença constante nos arrastos, o que ressalta o risco que essa pescaria impõe à ictiofauna local. Por outro lado, a incidência do arrasto sobre a atividade reprodutiva não está relacionada ao grau de frequência das espécies nas pescarias. Quatro daquelas de ocorrência constante (C. nobilis, $L$. laevigatus, $L$. breviceps e $M$. americanus) não foram registradas em reprodução, o que pode dever-se a (i) local e profundidade de desova diferentes daqueles onde atua a frota camaroeira, (ii) tamanho relativamente pequeno com que os indivíduos ocorrem nos arrastos, e/ou (iii) os adultos apresentarem baixa vulnerabilidade ao arrasto de fundo. Mas é significativo constatar-se que $30 \%$ das espécies que nas amostras tiveram ocorrência apenas acidental (dentre elas $C$. undecimalis e $O$. ruber) integram o arrasto com indivíduos em reprodução.

A elevada representatividade da família Sciaenidae, uma dentre cinco espécies de peixes desembarcadas, é reconhecida no litoral sul do Brasil (Bail \& Branco 2003, Gomes \& Chaves 2006, Souza \& Chaves 2007). Tal dominância decorre da família ser comum em águas rasas da região, dispondo-se sobre fundos de areia ou lama (Menezes \& Figueiredo 1980). São áreas onde também o camarão sete-barbas se distribui, inclusive integrando a dieta de vários Sciaenidae (Chaves \& Umbria 2003).

Os atributos que levam uma espécie a ser explotada relacionam-se diretamente à sua abundância e capturabilidade, mas outra variável concerne seu ciclo de vida. Aquelas com tendência k- estrategista têm maior risco de comprometimento do estoque que as com tendência r-estrategista (Alverson et al. 1994). Assim, a presença de raias, peixes tipicamente k-estrategistas, alerta para o potencial de prejuízo causado à ictiofauna pelo arrasto camaroeiro na região, ainda que apenas Rhinobatos percellens apresentasse indivíduos em atividade reprodutiva e junto com $Z$. brevirostris foram acidentais, e $N$. brasiliensis teve ocorrência acessória em relação aos de teleósteos.

Atividade reprodutiva foi registrada em todas as estações amostradas, mas a primavera de 2005, o outono de 2006 e o verão de 2007 destacaram-se pela presença de espécies em atividade reprodutiva intensa e muito intensa. Em região mais ao sul (Enseada, município de São Francisco do Sul), no mesmo período de estudo, Souza \& Chaves (2007) registraram na primavera de 2005 e no verão de 2007 maior número de espécies em atividade reprodutiva muito intensa, todavia com semelhanças e diferenças em relação ao presente trabalho. Em $P$. harroweri, por exemplo, a atividade reprodutiva foi muito intensa no verão de 2007 em ambas as regiões; em $S$. tesselatus o pico de atividade reprodutiva foi registrado no outono no presente trabalho e no verão de 2007 no de Souza \& Chaves (op. cit.). As dessemelhanças podem ser explicadas pelas especificidades de cada frota arrasteira quanto à área de operação e profundidade de atuação, que é maior em Enseada.

Comparando-se os verões de 2006 e 2007, observou-se que no segundo a pesca de arrasto incidiu sobre apenas uma espécie a mais em atividade reprodutiva intensa que no primeiro. Assim, o fato do período de defeso ter, em 2005, ocorrido no outono, enquanto em 2006, antecedido imediatamente o verão de 2007, não trouxe diferenças entre os dois verões na intensidade de atividade reprodutiva dos peixes. Essa constatação difere daquela apresentada por Souza \& Chaves (2007), que propuseram na área de pesca de Enseada que a interdição de arrasto antecedendo o verão pode ser favorável ao processo reprodutivo da ictiofauna.

Souza \& Chaves (2007) remetem à questão: é mais prejudicial retirar-se da população indivíduos jovens ou indivíduos adultos em reprodução? Ao 
estudarem a ictiofauna acompanhante da pesca camaroeira na região de Enseada, próxima a área do presente estudo, constaram que ambas as situações ocorrem com a mesma intensidade. Em Itapema do Norte, $45 \%$ das espécies apresentaram pelo menos um indivíduo em reprodução. Esta questão está longe de ser respondida, mas observa-se que independente do período analisado a pesca do arrasto de camarão sempre vai incidir sobre uma parcela da ictiofauna em reprodução, com menor intensidade no inverno (Souza \& Chaves op cit.).

\section{AGRADECIMENTOS}

Aos pescadores da comunidade de Itapema do Norte - SC pelo apoio prestado à realização do trabalho. Ao CNPq pelo auxílio financeiro (Edital Universal 2006) e bolsa ao segundo autor, e à CAPES pela bolsa à primeira autora.

\section{LITERATURA CITADA}

AGOSTINHO, AA, V MENDES, HI SUZUKI \& C CANZI. 1993 Avaliação da atividade reprodutiva da comunidade de peixes dos primeiros quilômetros a jusante do reservatório de Itaipu. UNIMAR, 15:175- 189.

ALVERSON, DL, MH FREEBERG, JG POPE \& SA MURAWSKI. 1994.A global assessment of fisheries by catch and discards. Roma, FAO Fisheries Technical Paper 339. 233p.

ARENDSE, CJ, A GOVENDER \& GM BRANCH. 2007. Are closed fishing seasons an effective means of increasing reproductive output? A per-recruit simulation using the limpet Cymbula granatina as a case history. Fisheries Research, 85(1-2):93100.

BAIL, GC \& JO BRANCO. 2003. Ocorrência, abundância e diversidade da ictiofauna na pesca do camarão sete-barbas, na região de penha, SC. Notas técnicas da FACIMAR, 7:73-82.

BRANCO, JO \& JR VERANI. 2006 A. Análise quali-quantitativa da ictiofauna acompanhante na pesca do camarão sete-barbas na Armação do Itapocoroy, Penha, Santa Catarina. Revista Brasileira de Zoologia, 23(2):381-391.

CHAVES, PT, G COVA-GRANDO \& C CALLUF. 2003. Demersal ichthyofauna in a continental shelf region on the south coast of Brazil exposed to shrimp trawl fisheries. Acta Biológica Paranaense, 32:69-82.

CHAVES, PT \& SC UMBRIA. 2003. Mudanças na dieta de peixes que se deslocam entre dois sistemas costeiros, estuário e Plataforma Continental. Braz. Arch. Biol. Tech., Curitiba, 46(1):41-46.

DAJOZ, R. 1983. Ecologia geral. Petrópolis, Vozes. 472p.

GOMES, ID \& PT CHAVES. 2006. Ictiofauna integrante da pesca de arrasto camaroeiro no litoral sul do Estado do Paraná, Brasil. Bioikos, 20(1): 9-13.

ISAAC, VJ, AS MARTINS, M HAIMOVICI, JP CASTELLO \& JM ANDRIGUETO-FILHO. 2006. Síntese do estado de conhecimento sobre a pesca marinha e estuarina do Brasil. In: ISAAC, VJ, AS MARTINS, M HAIMOVICI \& JM ANDRIGUETTO (Ed.). A pesca marinha e estuarina do Brasil no início do século XXI. Belém, Editora UFPA.

MENEZES, NA \& JL FIGUEIREDO. 1980. Manual de peixes marinhos do sudeste do Brasil. Museu de Zoologia da USP. IV. Teleostei (3). São Paulo, Museu de Zoologia da USP. 96p.

ROBERT, MC \& PT CHAVES. 2006. Dinâmica da atividade pesqueira artesanal em duas comunidades da região litorânea limítrofe Santa Catarina - Paraná, Brasil. Boletim do Instituto de Pesca, São Paulo, 32(1):15-23.

SOUZA, LM \& PT CHAVES. 2007. Atividade reprodutiva de peixes (Teleostei) e o defeso da pesca de arrasto no litoral norte de Santa Catarina, Brasil. Revista Brasileira de Zoologia 24(4):1113-1121.

SUNYÉ, PS. 2006. Diagnóstico da pesca no litoral do Estado de Santa Catarina. In: ISAAC, VJ, AS MARTINS, M. HAIMOVICI \& JM ANDRIGUETTO (Ed.). A pesca marinha e estuarina do Brasil no início do século XXI. Belém, Editora UFPA.

VAZZOLER, AEA de M. 1996. Biologia da reprodução de peixes teleósteos: teoria e prática. Maringá, EDUEM - SBI. 169p. 